ISSN 0103-9954

\title{
PROPRIEDADES FÍSICO-MECÂNICAS DE PAINÉIS AGLOMERADOS PRODUZIDOS COM DIFERENTES PROPORÇÕES DE MADEIRA E CASCA DE ARROZ ${ }^{1}$
}

\author{
PROPERTIES OF WOOD AND RICE HUSK PARTICLEBOARD IN DIFFERENT PROPORTIONS
}

\author{
Rafael Rodolfo de Melo ${ }^{2}$ Elio José Santini ${ }^{3}$ \\ Clovis Roberto Haselein ${ }^{4}$ Diego Martins Stangerlin ${ }^{5}$
}

\section{RESUMO}

No presente trabalho, avaliaram-se as propriedades físico-mecânicas de painéis aglomerados produzidos com diferentes proporções de madeira (Eucalyptus grandis) e casca de arroz. Para caracterização dos painéis produzidos com os adesivos ureia-formaldeído e tanino-formaldeído, nas proporções $0,20,40$, 60, 80 e 100\% de casca de arroz, determinaram-se as propriedades físicas (massa específica; teor de umidade; absorção de água e inchamento em espessura após 2 e 24 horas de imersão em água), e de resistência mecânica (flexão estática; ligação interna e arrancamento de parafusos). Os resultados obtidos indicaram que o acréscimo da casca de arroz proporcionou uma maior instabilidade e uma menor resistência das chapas. Quando confeccionados utilizando a resina à base de tanino, os painéis apresentaram qualidade superior.

Palavras-chave: painéis reconstituídos; casca de arroz; tanino-formaldeído; ureia-formaldeído.

\section{ABSTRACT}

This work aimed at evaluating the quality of particleboard manufactured with different wood proportions (Eucalyptus grandis) and rice husk. The composites were produced in the proportions 0, 20, 40, 60,80 , and $100 \%$ of rice husk with the use of urea-formaldehyde and tannin-formaldehyde adhesives. In order to characterize the quality of the particleboards, physical properties (density; moisture content; water absorption and thickness swelling after 2 and 24 hours of immersion in water) and mechanical properties (static bending; internal bonding and screw withdrawal) were considered. Results showed that increasing rice husk proportion caused larger instability and a decrease in the resistance of particleboards. Particleboards manufactured with tannin-formaldehyde resins presented higher quality when compared to ureaformaldehyde.

Keywords: particleboard; rice husks; tannin-formaldehyde; urea-formaldehyde.

\section{INTRODUÇÃO}

As chapas aglomeradas podem ser produzidas partindo de qualquer material ligno-celulósico que proporcionem resistência mecânica satisfatória com massa específica pré-estabelecida. Além da madeira, estudos apontam a viabilidade de utilização de outras fontes de fibras na manufatura de painéis aglomerados, como bagaço de cana-de-açúcar (OKINO et al., 1997; TEIXEIRA et al., 1997), bambu (CALEGARI et al., 2007), aparas de papel reciclado (CALEGARI et al., 2004), palha e casca de arroz (HIZIROGLU et al., 2005; NDAZI et al., 2006; MELO,2009), entre outros. Entretanto, a qualidade final do produto, pode ser limitada pela escolha do material. Dentre esses resíduos, aquele gerado no processo de beneficiamento do arroz apresenta um grande potencial sobretudo, em relação à disponibilidade de matéria-prima.

1. Parte da Dissertação de Mestrado do primeiro autor, apresentada ao Programa de Pós-Graduação em Engenharia Florestal da Universidade Federal de Santa Maria, Av. Roraima, 1000, CEP: 97105-900, Santa Maria (RS).

2. Engenheiro Florestal, MSc., Doutorando em Ciências Florestais, Universidade de Brasília, Faculdade de Tecnologia, Programa de Pós-Graduação em Ciências Florestais, Caixa Postal 04357, CEP: 70919-970, Brasília (DF).rrmelo2@yahoo.com.br

3. Engenheiro Florestal, Dr., Professor Associado do Departamento de Ciências Florestais, Centro de Ciências Rurais, Universidade Federal de Santa Maria, Av. Roraima, 1000, CEP: 97105-900, Santa Maria (RS). eliosantini@smail.ufsm.br

4. Engenheiro Florestal, PhD., Professor Associado do Departamento de Ciências Florestais, Centro de Ciências Rurais, Universidade Federal de Santa Maria, Av. Roraima, 1000, CEP: 97105-900, Santa Maria (RS). clovis.haselein@smail.ufsm.br

5. Engenheiro Florestal, MSc., Doutorando em Ciências Florestais, Universidade de Brasília, Faculdade de Tecnologia, Programa de Pós-Graduação em Ciências Florestais, Caixa Postal 04357, CEP: 70919-970, Brasília (DF). diego_stangerlin@yahoo.com.br

Recebido para publicação em 18/05/2009 e aceito em 20/09/2009. 
O cultivo do arroz ocupa o segundo lugar em área plantada no mundo, ficando atrás apenas do trigo. O maior produtor é a China, seguida da Índia, Indonésia, Bangladesh, Vietnã e outros. O Brasil ocupa o décimo lugar, com cerca de $2 \%$ da produção mundial (Instituto Brasileiro de Geografia e Estatística - IBGE, 2005). Logo, painéis que apresentam em sua constituição casca de arroz surgem como alternativa para um dos graves problemas do setor agrícola.

Chen (1980) destaca que, economicamente, a utilização da casca de arroz na confecção de painéis aglomerados pode ser viável, pois os custos para o transporte da casca de arroz são competitivos com os custos para o transporte de partículas de madeira. A aquisição, no entanto, da casca de arroz pode sair mais barata, por se tratar de um resíduo agrícola que, na maioria das vezes, não é aproveitado. Já a madeira utilizada na produção de partículas poderia ser utilizada para manufatura de produtos de maior valor agregado.

Por ser de difícil decomposição e pelo alto percentual de sílica que possui, esse resíduo constitui um problema ambiental e de saúde, sobretudo nas regiões onde o cultivo do arroz se faz em larga escala. A partir do momento em que esse material for incorporado em um processo produtivo como uma matéria-prima alternativa a manufatura de painéis, ele será valorizado e deixará de ser um resíduo. Essa tecnologia da fabricação de painéis aglomerados utilizando a casca de arroz vem ganhando um importante enfoque, especialmente nos países asiáticos, que são os principais produtores de arroz.

O Rio Grande do Sul produz um volume expressivo desse resíduo na metade sul do Estado, o que facilita a logística de sua utilização. Estima-se que em torno de $40 \%$ do volume nacional de arroz é produzido no Estado, o que resultaria, em aproximadamente, um milhão de toneladas de casca de arroz anualmente. Uma fábrica de chapas aglomeradas de médio porte consome de 150 a 200 mil toneladas de matéria-prima (madeira) anualmente. Então, esse volume seria suficiente para suprir a demanda de mais de uma unidade industrial. Desse modo, busca-se desenvolver formas de aproveitamento desse resíduo na fabricação de chapas aglomeradas, para evitar ou diminuir o problema ambiental e, ao mesmo tempo, servir como mais uma fonte de renda para região (MELO, 2009). Nesse sentido, o presente trabalho teve como objetivo avaliar o efeito da adição, em diferentes proporções, de casca de arroz em painéis aglomerados constituídos com madeira de Eucalyptus grandis W. Hill ex Maiden, e suas implicações nas propriedades físicas e de resistência mecânica.

\section{MATERIAL E MÉTODOS}

\section{Coleta e preparo da matéria-prima}

Para manufatura dos painéis foram utilizadas partículas da madeira de Eucalyptus grandis W. Hill ex Maiden e cascas de arroz (Oryza Sativa L.). Seis árvores foram obtidas em povoamentos florestais homogêneos de aproximadamente 15 anos, localizados no Campus da Universidade Federal de Santa Maria, enquanto que a casca de arroz foi coletada no engenho de beneficiamento de arroz da Cooperativa de Arrozeiros Camobi, localizado em Santa Maria, RS.

Das árvores, foram retiradas tábuas as quais foram resserradas com cortes perpendiculares a grã, obtendo-se blocos de $5 \mathrm{~cm}$ de espessura (sentido tangencial), $7 \mathrm{~cm}$ de comprimento (sentido longitudinal) e largura variável (sentido radial), conforme o diâmetro e a posição de obtenção das tábuas na tora. Os blocos foram transformados em flocos em um moinho de facas, e estes, em partículas, ao serem processados em um moinho de martelos utilizando peneira com malha de $8 \mathrm{~mm}$ em diâmetro. As cascas de arroz também foram processadas em moinho de martelos, mas utilizando uma peneira com malha de $6 \mathrm{~mm}$. Em seguida, foram removidos os finos utilizando-se uma peneira com malha de $1,0 \times 1,0 \mathrm{~mm}$.

\section{Manufatura dos painéis}

Na confecção dos painéis foi utilizada uma resina comercial à base de ureia-formaldeído (UF) e, produzida uma resina a base de tanino-formaldeído (TF), utilizando o extrato em pó do tanino da Acacia mearnsii De Wild. Para a produção, o extrato em pó foi diluído em água (1:1), na qual permaneceu por 24 horas para hidratação. Posteriormente, foi adicionado hidróxido de sódio $(\mathrm{NaOH})$ até que o $\mathrm{pH} 8$ fosse atingido. O formaldeído foi adicionado apenas no momento da aplicação do adesivo, na proporção de $10 \%$ do teor de sólidos da resina produzida.

A composição dos painéis foi pré-estabelecida de modo que apresentassem $91 \%$ de partículas, $8 \%$ de 
adesivo e $1 \%$ de parafina, com massa específica de $0,65 \mathrm{~g} / \mathrm{cm}^{3}$. A pré-determinação da massa específica foi baseada no peso seco das partículas, no teor de sólidos do adesivo e da parafina utilizada. Os parâmetros utilizados para a produção foram: força de prensagem $30 \mathrm{kgf} / \mathrm{cm}^{2}$; teor de umidade do colchão de $12 \%$ para UF e de $20 \%$ para TF; tempo de prensagem 8 minutos para UF e 10 minutos para TF e; tempo de fechamento da prensa de 40 segundos. As variações do teor de umidade do colchão influíram, consequentemente, no tempo de prensagem. Tais alterações seguiram recomendações de Hillig et al. (2002), que observou melhores características de painéis produzidos com TF quando aumentado o teor de umidade do colchão.

As chapas foram produzidas utilizando seis diferentes proporções de madeira e casca de arroz para cada adesivo (Tabela 1). Ao todo, foram confeccionados 72 painéis aglomerados, seis para cada tratamento, com as dimensões de $50 \times 50 \times 0,95 \mathrm{~cm}$, todos confeccionados com massa específica nominal de $0,65 \mathrm{~g} / \mathrm{cm}^{3}$.

TABELA 1: Composições dos painéis com as proporções entre madeira e casca de arroz.

TABLE 1: Particleboard composition with wood and rice husk.

\begin{tabular}{|c|c|c|c|c|}
\hline Identificação & Adesivo & Repetições & Madeira & Casca de Arroz \\
\hline $0 \mathrm{C}-\mathrm{UF}$ & \multirow{6}{*}{$\begin{array}{l}\text { Ureia-Formaldeído } \\
\text { (UF) }\end{array}$} & 6 & 100 & 0 \\
\hline $20 \mathrm{C}-\mathrm{UF}$ & & 6 & 80 & 20 \\
\hline $40 \mathrm{C}-\mathrm{UF}$ & & 6 & 60 & 40 \\
\hline $60 C-U F$ & & 6 & 40 & 60 \\
\hline $80 \mathrm{C}-\mathrm{UF}$ & & 6 & 20 & 80 \\
\hline $100 \mathrm{C}-\mathrm{UF}$ & & 6 & 0 & 100 \\
\hline $0 \mathrm{C}-\mathrm{TF}$ & \multirow{6}{*}{$\begin{array}{c}\text { Tanino- } \\
\text { Formaldeído (TF) }\end{array}$} & 6 & 100 & 0 \\
\hline $20 \mathrm{C}-\mathrm{TF}$ & & 6 & 80 & 20 \\
\hline $40 \mathrm{C}-\mathrm{TF}$ & & 6 & 60 & 40 \\
\hline $60 \mathrm{C}-\mathrm{TF}$ & & 6 & 40 & 60 \\
\hline $80 \mathrm{C}-\mathrm{TF}$ & & 6 & 20 & 80 \\
\hline $100 \mathrm{C}-\mathrm{TF}$ & & 6 & 0 & 100 \\
\hline
\end{tabular}

\section{Ensaios realizados e análise dos resultados}

De cada painel, foram retiradas amostras para determinação das propriedades físicas (teor de umidade; massa específica; absorção d'água e inchamento em espessura após 2 e 24 horas de imersão em água) e mecânicas (flexão estática; resistência ao arrancamento de parafusos e ligação interna). Os ensaios foram realizados adotando-se as recomendações da ASTM D 1037 (1998).

Os dados das propriedades físico-mecânicas foram avaliados por análise de regressão. Para isso, foi realizada a modelagem dos parâmetros com o auxílio do pacote estatístico Satistical Analysis System - SAS ${ }^{\circledR}$ (1998), com as variáveis avaliadas em função do percentual de casca nos painéis (PC). Para todos os modelos gerados foram avaliadas as condicionantes de regressão, independência dos dados, normalidade e homogeneidade de variância, pelos testes de Durbin Watson, Shapiro Wilk e Withe, respectivamente. Quando não satisfeitas as duas últimas condicionantes, foi realizada a transformação dos dados, conforme recomendado por Schneider et al. (2009).

\section{RESULTADOS E DISCUSSÃO}

\section{Propriedades físicas}

\section{Teor de umidade}

Após serem colocadas em estufa a $60^{\circ} \mathrm{C}$ por 24 horas, as partículas de madeira e casca de arroz apresentaram, em média, $3,21 \pm 0,24 \%$ e $2,97 \pm 0,37 \%$ respectivamente, de teores de umidade. Para fins práticos, o teor de umidade das partículas, no momento da manufatura dos painéis foi considerado para ambos os casos como 3\%. Quanto ao teor de umidade do colchão (Tabela 2), para os tratamentos com ureiaformaldeído, pré-estabelecido em 12\%, os resultados oscilaram entre 11,21 e 13,09\%, e para taninoformaldeído, pré-estabelecido em $20 \%$, a variação ficou entre 19,40 e $21,62 \%$. Vários fatores podem ter influenciado nessa variação, um deles é própria diferença de umidade das partículas ao saírem da estufa. Outra influência deve-se às pequenas parcelas dos produtos aplicados (água, adesivo e parafina) que são perdidas por aderirem nos instrumento (mangueira, pistola e tambor misturador). Por esses motivos, Hillig et al. (2002) destacam que pequenas variações nesse parâmetro são comuns, por se tratar de uma variável de 
difícil controle.

TABELA 2: Valores de massa específica observada (MEo), teor de umidade de colchão (TUc) e teor de umidade de equilíbrio (TUeq) dos painéis.

TABLE 2: Results of density observed (MEo), mat moisture content (TUc) and equilibrium moisture content (TUeq) of the particleboards.

\begin{tabular}{|c|c|c|c|c|c|}
\hline \multirow{2}{*}{ Tratamento } & \multicolumn{2}{|c|}{ Partículas (\%) } & \multirow{2}{*}{$\begin{array}{c}\text { MEo } \\
\left(\mathrm{g} / \mathrm{cm}^{3}\right)\end{array}$} & \multirow{2}{*}{$\begin{array}{l}\text { TUc } \\
(\%)\end{array}$} & \multirow{2}{*}{$\begin{array}{c}\text { TUeq }^{1} \\
(\%)\end{array}$} \\
\hline & Madeira & Casca & & & \\
\hline $0 \mathrm{C}-\mathrm{UF}$ & 100 & 0 & 0,66 & 12,54 & 8,32 \\
\hline $20 \mathrm{C}-\mathrm{UF}$ & 80 & 20 & 0,67 & 12,47 & 8,39 \\
\hline $40 \mathrm{C}-\mathrm{UF}$ & 60 & 40 & 0,67 & 13,09 & 8,29 \\
\hline $60 \mathrm{C}-\mathrm{UF}$ & 40 & 60 & 0,64 & 12,34 & 8,41 \\
\hline $80 \mathrm{C}-\mathrm{UF}$ & 20 & 80 & 0,63 & 11,21 & 8,49 \\
\hline $100 \mathrm{C}-\mathrm{UF}$ & 0 & 100 & 0,63 & 11,89 & 8,39 \\
\hline $0 \mathrm{C}-\mathrm{TF}$ & 100 & 0 & 0,66 & 19,40 & 8,64 \\
\hline $20 \mathrm{C}-\mathrm{TF}$ & 80 & 20 & 0,68 & 21,62 & 8,52 \\
\hline $40 \mathrm{C}-\mathrm{TF}$ & 60 & 40 & 0,67 & 19,94 & 8,37 \\
\hline $60 \mathrm{C}-\mathrm{TF}$ & 40 & 60 & 0,66 & 21,06 & 8,50 \\
\hline $80 \mathrm{C}-\mathrm{TF}$ & 20 & 80 & 0,64 & 20,79 & 8,55 \\
\hline $100 \mathrm{C}-\mathrm{TF}$ & 0 & 100 & 0,64 & 19,68 & 8,42 \\
\hline
\end{tabular}

Em que: ${ }^{1}$ Obtido em câmara climática com temperatura de $20^{\circ} \mathrm{C}$ e umidade relativa do ar de $65 \%$.

O teor de umidade de equilíbrio médio das amostras variou entre 8,39 e 8,64\% (Tabela 2 e Figura 1). Percebe-se que o acréscimo da casca de arroz nos painéis confeccionados não influenciou na umidade de equilíbrio desses painéis. Todos os tratamentos mostraram um padrão de variação semelhante, não sendo observadas diferenças marcantes entre eles. Essa baixa variabilidade proporciona uma maior confiabilidade nos demais resultados obtidos, já que a umidade do painel pode interferir significativamente em suas propriedades.

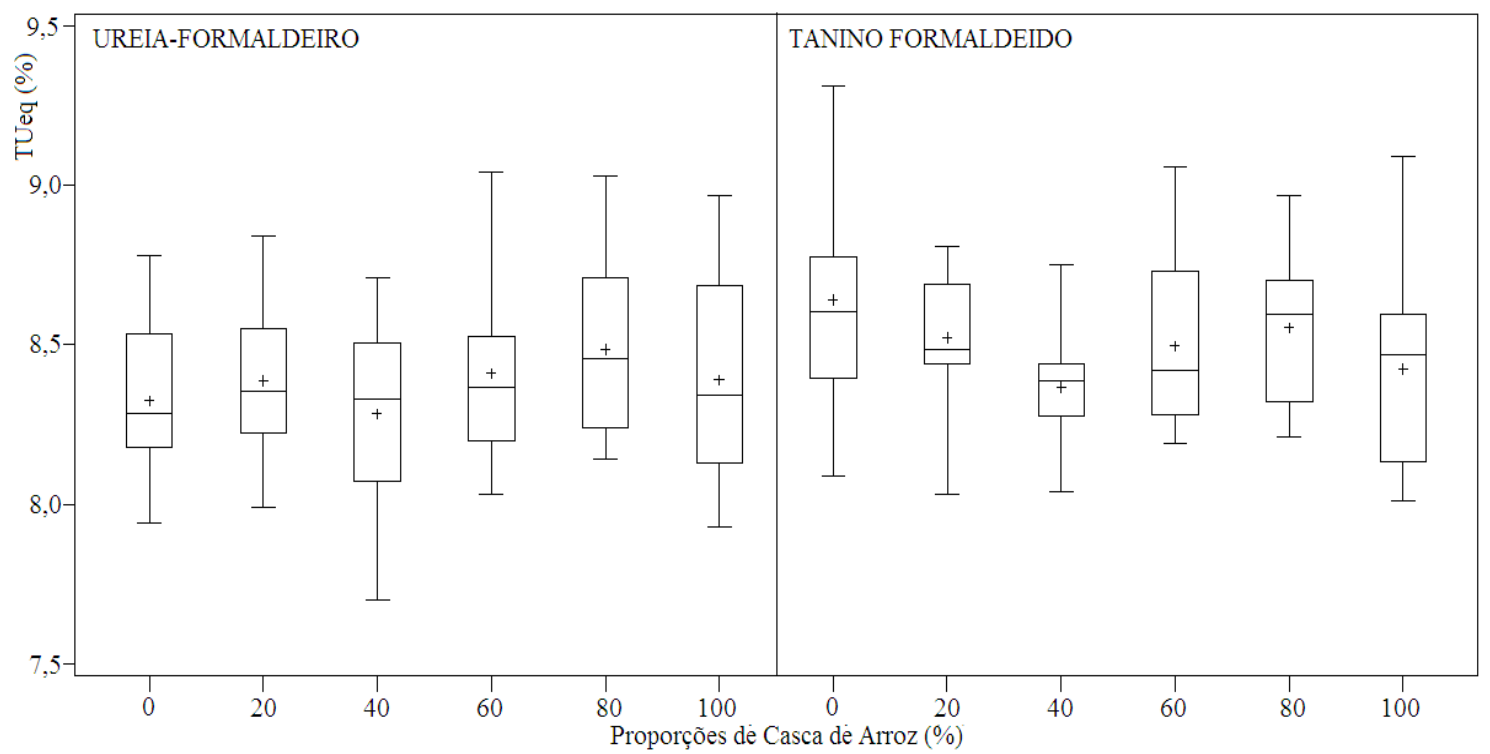

FIGURA 1: Variação do teor de umidade de equilíbrio dos painéis.

FIGURE 1: Equilibrium moisture content variation the particleboard.

\section{Massa específica}

A massa específica básica média observada para a madeira de Eucalyptus grandis foi de $0,51 \pm 0,04$ $\mathrm{g} / \mathrm{cm}^{3}$. Esses resultados foram superiores aos observados por Ferreira (1997) e Lopes et al. (2004), que encontraram para a mesma espécie, $0,43 \mathrm{~g} / \mathrm{cm}^{3}$ e $0,46 \mathrm{~g} / \mathrm{cm}^{3}$ respectivamente. Já Ciniglio (1998) encontrou 
$0,57 \mathrm{~g} / \mathrm{cm}^{3}$, valor superior ao desse estudo. Essa variabilidade nos valores de massa específica para uma mesma espécie pode ser atribuída à idade dos indivíduos, fatores ambientais ou genéticos (TOMAZELLO FILHO, 1987).

A massa específica média determinada ao teor de umidade de equilíbrio variou entre 0,63 e 0,67 $\mathrm{g} / \mathrm{cm}^{3}$ para os painéis confeccionados com ureia-formaldeído, e entre 0,64 a $0,68 \mathrm{~g} / \mathrm{cm}^{3}$, para aqueles confeccionados com tanino-formaldeído (Tabela 2), tendo, em ambos os casos, resultados relativamente próximos aos pré-estabelecidos pela massa específica nominal $\left(0,65 \mathrm{~g} / \mathrm{cm}^{3}\right)$.

Variações de massa específica foram observadas entre tratamentos, entre painéis de um mesmo tratamento e dentro de um mesmo painel (Figura 2). Essa pequena variação, também observada por Hillig et al. (2002) e Dacosta et al. (2005), ocorre no processo manual de manufatura dos painéis em laboratório, especialmente nas fases de montagem do colchão e/ou na adição de resinas e outros aditivos. Iwakiri (1989) relata ainda que o material utilizado para confecção dos painéis, o teor de umidade das partículas e as diferenças de massa específica entre partículas também podem proporcionar variações na massa específica final do painel. Tanto para os painéis produzidos com adesivos tânicos como para os produzidos com adesivos ureicos, foi observado que as chapas produzidas com maiores proporções de casca de arroz apresentaram valores de massa específica média inferiores aos produzidos com maior percentual de madeira.

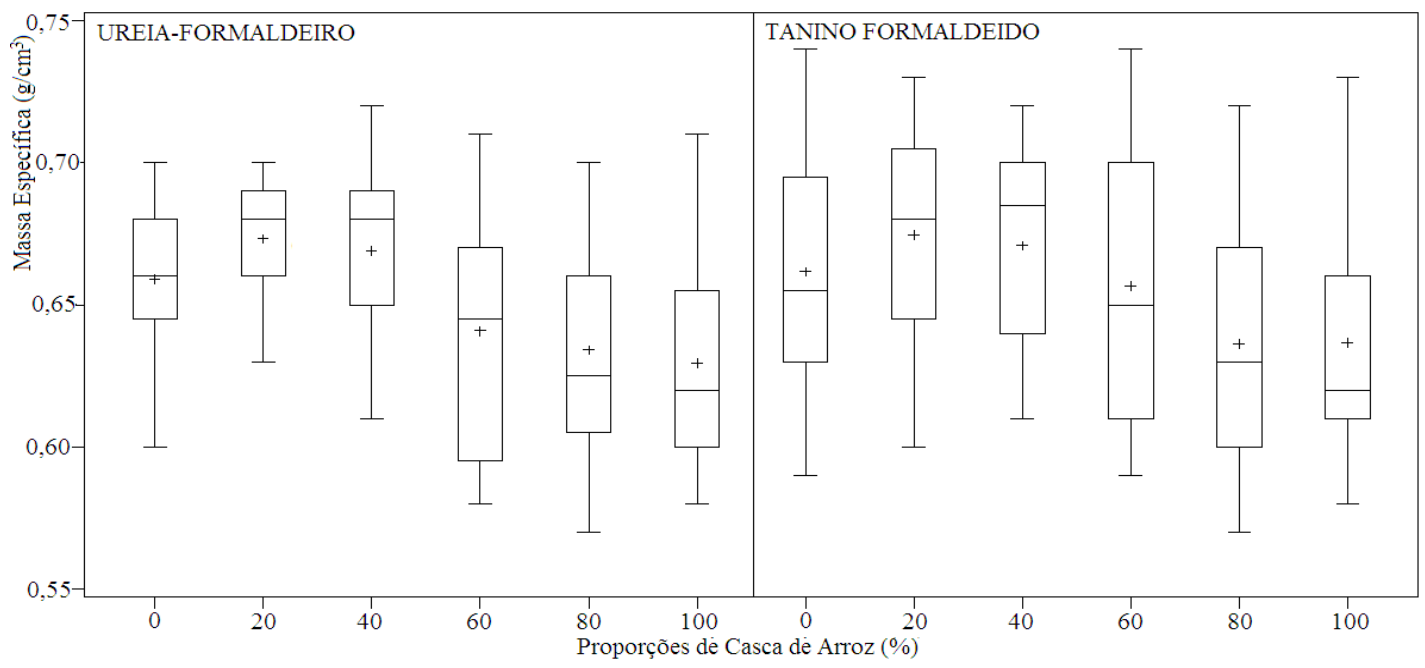

FIGURA 2: Variação da massa específica aparente dos painéis.

FIGURE 2: Density variation of the particleboard.

\section{Absorção d'água}

As equações ajustadas para absorção d'água durante 2 (Ab2) e 24 (Ab24) horas de imersão para os dois adesivos podem ser observadas na Figura 3. Para Ab2, por causa da visual superposição dos dados, foi realizada análise de covariância, a qual indicou ser não significativa a variação entre adesivos.

As equações modeladas apresentaram ajuste significativo, com o melhor evidenciado para Ab24 em painéis confeccionados com TF $\left(\mathrm{R}^{2}=0,71\right)$. Observa-se em todos os casos, que o aumento do percentual de casca nos painéis proporcionou os maiores valores de absorção de água, tanto em 2 como em 24 horas. Esse resultado foi diferente daquele observado por Pauleski et al. (2007), que verificou para outro gênero de painel, compósitos laminados manufaturados com polietileno de alta densidade (PEAD), que o aumento na proporção de casca de arroz em relação às partículas madeira de Eucalyptus grandis, diminuiu a absorção d'água e o inchamento em espessura dos painéis. Naquele trabalho a autora utilizou o PEAD como matriz, em altas proporções ( $65 \%$ ou mais). 


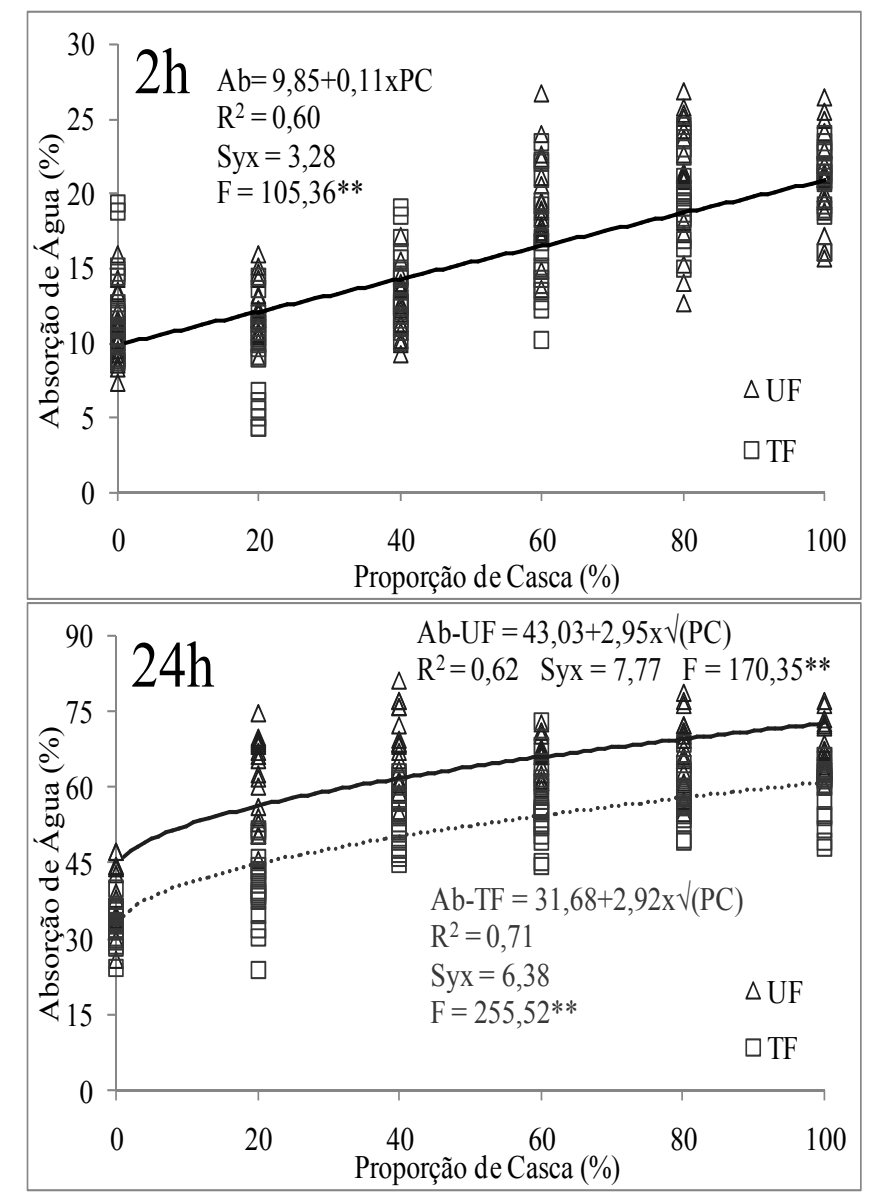

FIGURA 3: Absorção de água (Ab) por 2 e $24 \mathrm{~h}$ em função da proporção de casca de arroz para painéis produzidos com adesivos de ureia-formaldeído (UF) e tanino-formaldeído (TF)

FIGURE 3: Water absorption (Ab) in 2 and 24 hours as a function of rice husk percentage, for particleboard manufactured with urea-formaldehyde (UF) and tannin-formaldehyde (TF).

\section{Inchamento em espessura}

O ajuste das equações do inchamento em espessura durante 2 (IE2) e 24 (IE24) horas em função do percentual de casca para os diferentes adesivos pode ser observado na Figura 4. Embora tenham sido evidenciados ajustes significativos em todos os casos, não foi observado um bom ajuste $\left(R^{2}=0,18\right)$ para o IE24 em chapas que utilizaram UF. No geral, o aumento da proporção de casca nos painéis proporcionou maiores inchamentos, exceto para IE24-UF, para qual não foi verificada uma tendência clara. Hirizoglu et al. (2005) constataram que painéis produzidos com palha de arroz apresentam elevada absorção de água (Ab) e inchamento em espessura (IE), e que, quando misturados com madeira na mesma proporção, esses resultados foram significativamente reduzidos.

Considerando os valores médios de IE para o tipo de adesivo isoladamente, painéis confeccionados com TF apresentaram uma maior estabilidade dimensional, tanto para 2 como para 24 horas de imersão. Os taninos são ricos em fenóis e polifenóis e, quando misturados ao formaldeído, produzem, reconhecidamente, adesivos com boa resistência à umidade. No entanto, apesar de Pizzi et al. (1981) afirmarem que adesivos de tanino-formaldeído apresentam propriedades similares aos de fenol-formaldeído, a qualidade desses adesivos depende muito de sua formulação, do material de origem e de tratamentos para melhoria de sua eficiência. Geralmente, adesivos à base de tanino apresentam eficiência intermediária entre as resinas ureicas e fenólicas. 


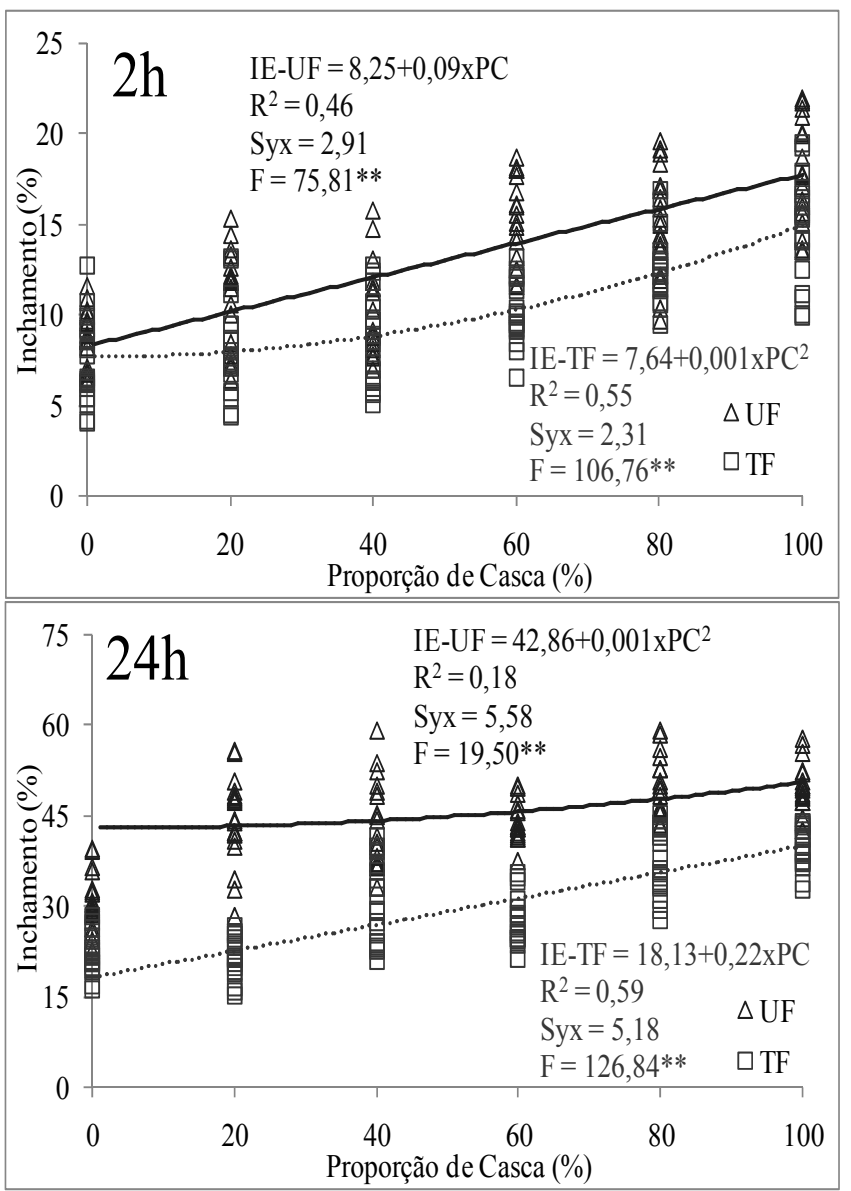

FIGURA 4: Inchamento em espessura (IE) por 2 e 24 horas em função da proporção de casca de arroz nos painéis produzidos com os adesivos ureia-formaldeído (UF) e tanino-formaldeído (TF).

FIGURE 4: Thickness swelling (IE) in 2 and 24 hours as a function of rice husk percentage, for particleboard with urea-formaldehyde (UF) and tannin-formaldehyde (TF).

Mesmo nos tratamentos de maior estabilidade dimensional ( 0 e $20 \%$ de casca de arroz) os valores de inchamento foram superiores aos exigidos pela norma do German Standards Commitee (1971), que estabelece o inchamento máximo de $12 \%$ (fenol) ou $15 \%$ (ureia) às 24 horas. Resultados semelhantes foram observados por Okino et al. (1997), que evidenciaram para painéis confeccionados com bagaço de cana-deaçúcar, utilizando adesivos a base de ureia e tanino, que só foi possível atingir o valor especificado pela normativa quando as partículas foram modificadas quimicamente pelo processo de acetilação. No entanto, a norma de comercialização ANSI 208.1 (1987), considera aceitável até 35\% de IE às 24 horas, que foi atingida pelos painéis confeccionados exclusivamente com madeira para ambos os adesivos, e por aquelas coladas com TF com proporção de casca máxima de $60 \%$.

\section{Resistência Mecânica}

\section{Flexão estática}

A estimação do MOR em função do MOE apresentou um bom ajuste por regressão linear (Figura 5). Foi observada, ainda, uma sobreposição de tendências para os adesivos utilizados, a qual foi confirmada pela análise de covariância, indicando baixa variação entre os adesivos, podendo ser o modelo bem representado por uma única equação. A relação entre essas variáveis geralmente segue esse padrão e, segundo Kelly (1977), deve-se à influência que diversas variáveis de processamento exercem de forma similar tanto no MOE como no MOR. 


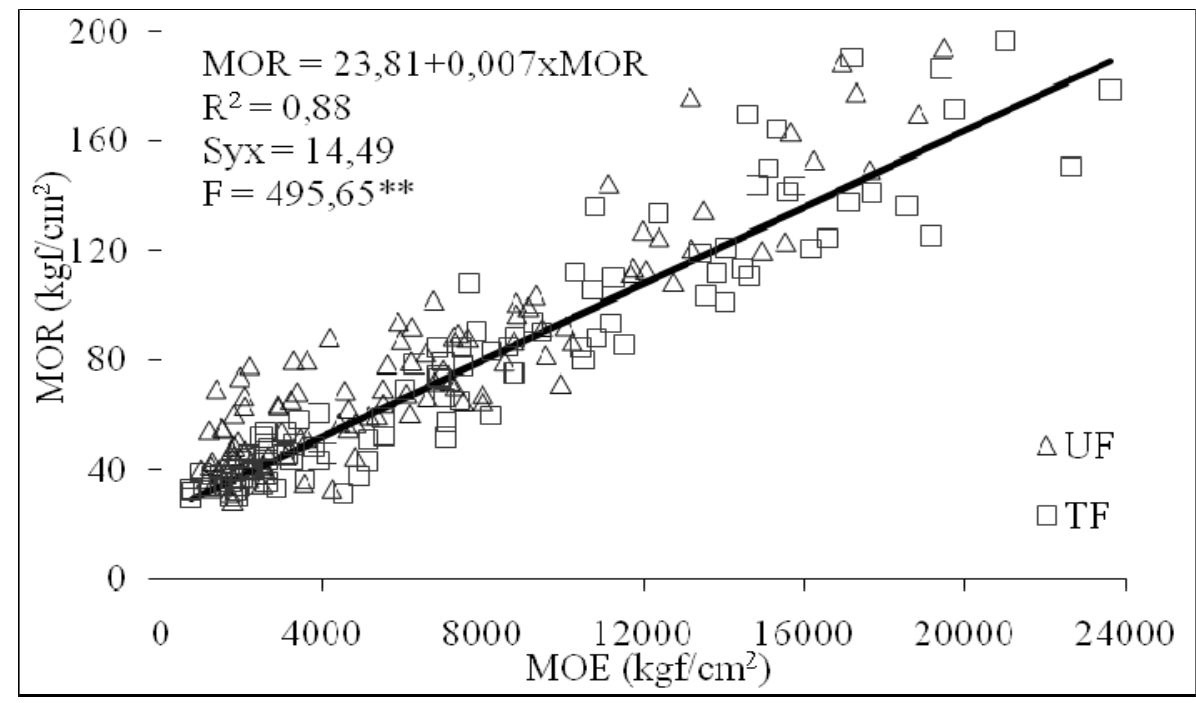

FIGURA 5: Módulo de ruptura (MOR) em função do módulo de elasticidade (MOE) para painéis produzidos com os adesivos ureia-formaldeído (UF) e tanino-formaldeído (TF).

FIGURE 5: Rupture modulus (MOR) as a function elasticity modulus (MOE), for particleboard with ureaformaldehyde (UF) and tannin-formaldehyde (TF).

As equações ajustadas para determinação do MOR e MOE em função do teor de casca para os painéis produzidos com UF e TF podem ser observadas na Figura 6. Para o MOE, foi detectada variação significativa entre os tipos de adesivos, já para o MOR a análise de covariância indicou não haver diferença para o tipo de adesivo utilizado, e assim, gerada uma única equação na predição dessa propriedade.

Todas as equações geradas apresentaram um elevado coeficiente de determinação e indicaram que o acréscimo da proporção de casca nos painéis promoveu a redução da sua resistência. Os resultados obtidos tanto para MOR como para o MOE apontaram uma redução com o aumento do percentual de casca. Outros pesquisadores, entre eles, Ajiwe et al. (1998), Han et al. (1998), Lee e Kang (1998) e Ndazi et al. (2006), também obtiveram resultados semelhantes ao deste estudo. Eles também observaram que o aumento da proporção de casca de arroz em painéis proporcionou diminuição dos parâmetros que indicam a resistência e a rigidez desses materiais. Hiziroglu et al. (2005), ao estudarem painéis produzidos com diferentes proporções de Eucalyptus camaldulensis, palha de arroz e bambu, observaram que a resistência mecânica de painéis mistos foi inferior, quando comparada aos painéis produzidos exclusivamente com partículas de Eucalyptus camaldulensis.

A norma americana de comercialização ANSI A 208.1 para painéis de média densidade $(0,60$ a 0,85 $\mathrm{g} / \mathrm{cm}^{3}$ ) do tipo 1, admite como valores mínimos requeridos os valores de $17.600 \mathrm{kgf} / \mathrm{cm}^{2}$ para o MOE e 112 $\mathrm{kgf} / \mathrm{cm}^{2}$ para o MOR (ANSI, 1987). Nesse sentido, pode-se observar que para o MOE, apenas painéis colados com tanino-formadeído e que utilizaram exclusivamente madeira para sua produção atingiram os valores requeridos. Quanto ao MOR, atenderam as exigências das especificações apenas chapas que não utilizaram casca de arroz em sua composição, confeccionadas com ambas as resinas analisadas. Todavia, para norma DIN 68761(1)-1961(3) (GERMAN STANDARDS COMMITEE, 1971) que não estabelece escalas para diferentes massas específicas, o valor mínimo exigido é de $180 \mathrm{kgf} / \mathrm{cm}^{2}$, que é superior a qualquer valor médio observado. Os resultados alcançados no presente estudo contradizem Hancock e Chandramouli (1974), que afirmam ser as propriedades dos painéis produzidos com casca de arroz similares às dos painéis com partículas de madeira. Entretanto inúmeras variáveis envolvidas no processo produtivo dos painéis, tais como adesivos, massa específica, tempo de prensagem, dentre outras, influenciam diretamente a qualidade do produto final e podem ter contribuído nesta divergência de resultados. Outra questão pode ter sido o fato das indústrias de painéis à base de madeira terem evoluído significativamente desde o período que fora desenvolvido aquele trabalho até hoje. 


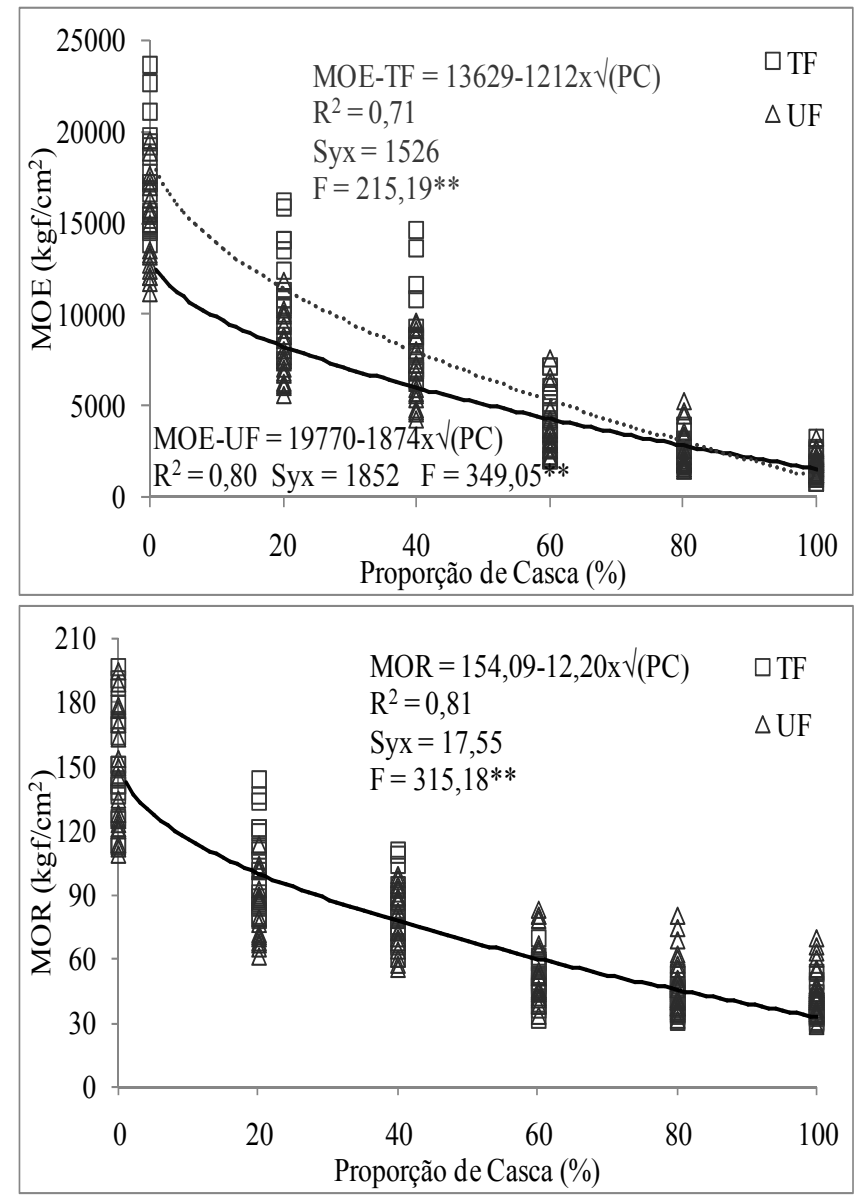

FIGURA 6: MOE e MOR em função da proporção de casca de arroz, para os adesivos ureia-formaldeído (UF) e tanino-formaldeído (TF).

FIGURE 6: MOE and MOR as a function rice husk percentage, for particleboard glued with ureaformaldehyde (UF) and tannin-formaldehyde (TF).

\section{Resistência ao arrancamento de parafusos}

As equações ajustadas para os valores de resistência ao arrancamento de parafusos (AP) em função do percentual de casca e para cada tipo de adesivo encontram-se na Figura 7. A análise de covariância avaliada para AP indicou não haver diferença entre os adesivos estudados para equação ajustada. Carneiro et al. (2004), ao estudarem diversas formulações de resinas a base de tanino e ureia, puras ou misturas com diferentes conposições, constataram que as variações entre adesivos não influenciaram a resistência ao arrancamento de parafuso para chapas de flocos de Pinus elliottii e Eucalyptus grandis. Pedrazzi et al. (2006), ao estudar agloremados confeccionados com resíduos de madeira e colados com resina à base de ureia-fomaldeído, observou que o aumento da quantidade de adesivo aplicado proporcionou maior resistência ao arracamento de parafusos. A equação ajustada em função do percentual de casca proporcionou um alto ajuste $\left(\mathrm{R}^{2}=0,89\right)$, indicando que acréscimo de casca de arroz nos painéis proporciona uma redução da resistência ao arrancamento de parafusos dos painéis. 


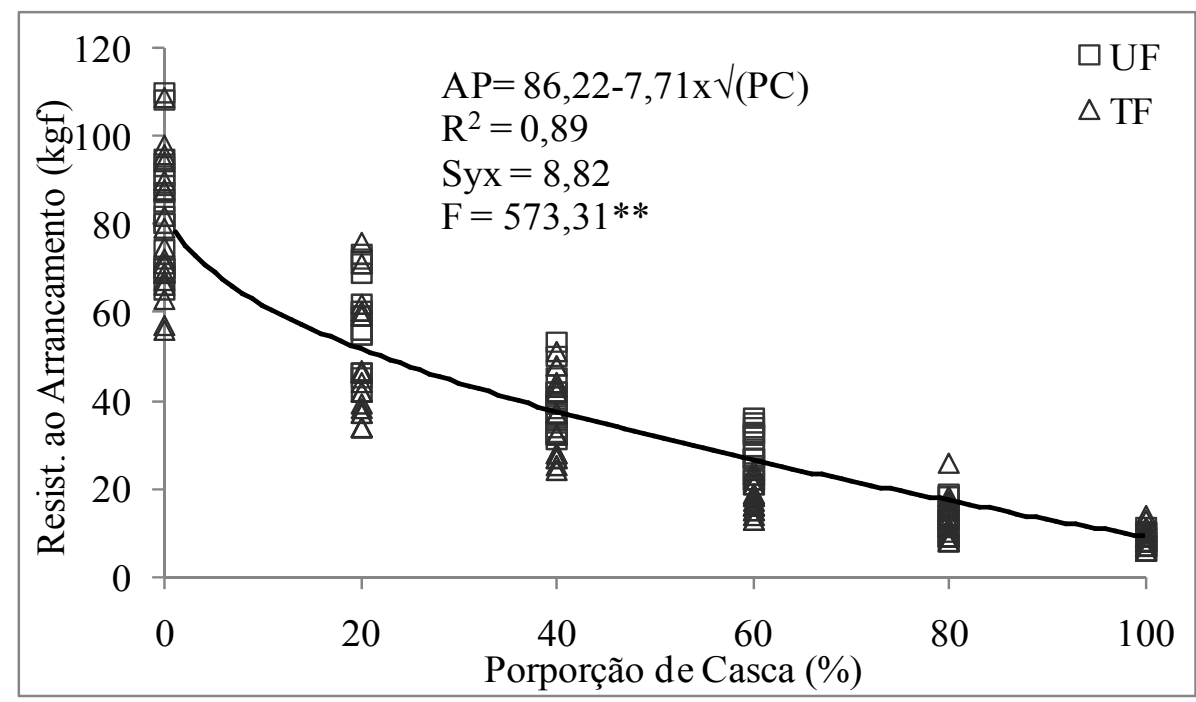

FIGURA 7: Resistência ao arrancamento de parafusos (AP) em função da proporção de casca de arroz, nos painéis para os dois adesivos utilizados.

FIGURE 7: Screw withdrawal (AP) as a function rice husk percentage, for two adhesives utilized.

Os valores mínimos requeridos pela norma ANSI A 208.1 (1987) de resistência ao arrancamento de parafusos para os tipos de painéis estudados são de $102 \mathrm{kgf}$. Apesar dos tratamentos que utilizaram apenas madeira para ambos os tipos de adesivo, terem apresentado valores acima dos exigidos por esta norma para algumas de suas amostras, dentre os valores médios, nenhum tratamento se apresentou satisfatório.

\section{Tração perpendicular à superfície do painel}

Também conhecida como ligação interna (LI), a partir desse ensaio, pode-se avaliar a adesão interna das partículas. A influência da proporção de casca na ligação interna dos painéis pode ser observada na Figura 8. Foram encontrados altos coeficientes de determinação $\left(R^{2}\right)$ nos modelos ajustados para ambas as resinas UF $\left(\mathrm{R}^{2}=0,84\right)$ e TF $\left(\mathrm{R}^{2}=0,83\right)$, o que indica que a LI das chapas pode ser facilmente prevista partindo da inclusão das partículas de casca de arroz.

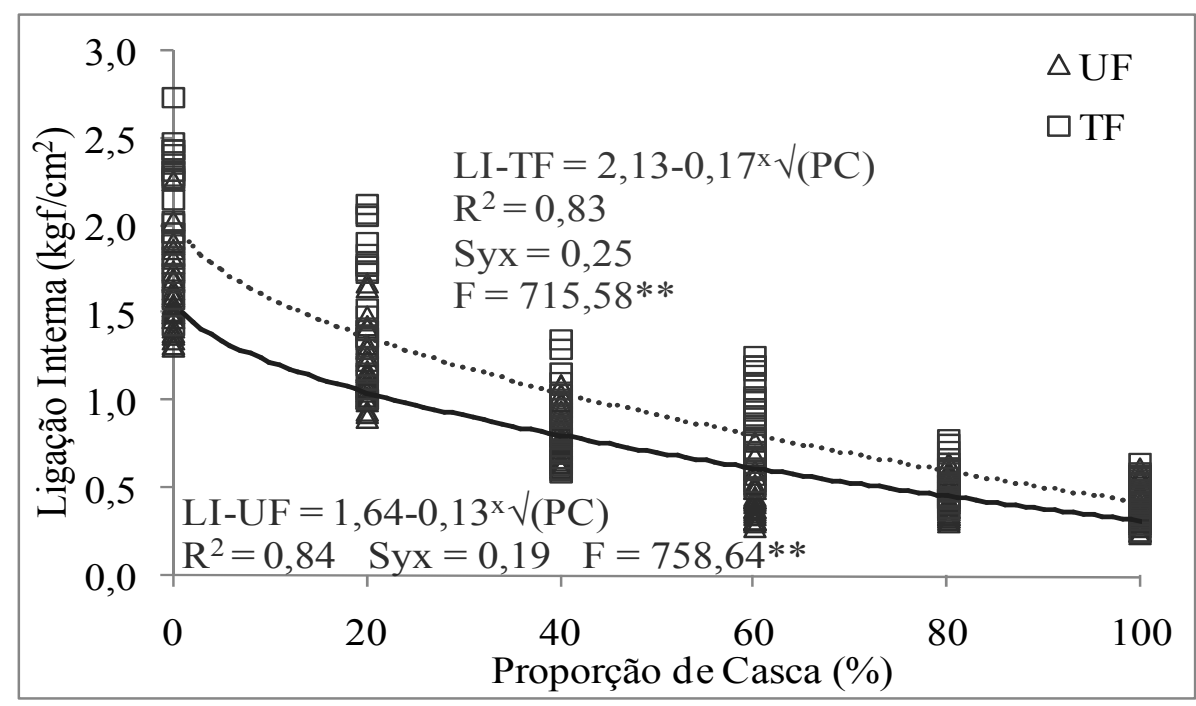

FIGURA 8: Ligação interna (LI) em função da proporção de casca, para os adesivos ureia-formaldeído (UF) e tanino-formaldeído (TF).

FIGURE 8: Internal bonding (LI) as a function rice husk percentage, for particleboard with ureaformaldehyde (UF) and tannin-formaldehyde (TF).

Para a ligação interna, as normas de comercialização americana (ANSI A 208.1, 1987) e alemã 
(GERMAN STANDARDS COMMITEE, 1971) estabelecem os valores mínimos de 4,22 e 3,50 kgf $/ \mathrm{cm}^{2}$ respectivamente. No presente estudo, os valores obtidos oscilaram entre 0,40 a $2,09 \mathrm{kgf} / \mathrm{cm}^{2}$, não sendo atendido para nenhuma normativa os valores mínimos exigidos.

A ligação interna foi uma das maiores deficiências das chapas que apresentavam uma maior proporção de casca de arroz. Essa característica, que indica o quão significativa foi à interação realizada entre as partículas e o adesivo utilizado, está diretamente relacionada com todas as demais propriedades físico-mecânicas avaliadas no presente estudo. Os baixos valores de ligação interna podem estar relacionados à estrutura cilíndrica e oca das partículas da palha, que podem ter atuado como uma barreira durante a aplicação da resina (HIZIROGLU et al., 2005). Outra provável influência é a baixa permeabilidade mencionada por Youngquist et al. (1993), que pode ter dificultado a colagem das partículas. Lee e Kang (1998) também observaram que o aumento do percentual das partículas de casca de arroz resulta numa diminuição das propriedades físicas e de resistência mecânica das chapas. Entretanto, o seu incremento pequenas concentrações, como de 5 a $15 \%$, podem não apresentar nenhum impacto significativo nas propriedades das chapas.

Ndazi et al. (2006) produziram chapas com casca de arroz e adesivo à base de tanino de Acacia mimosa. Segundo os autores, a casca de arroz está entre os resíduos agrícolas que estão disponíveis facilmente em grandes quantidades e nesse ponto de vista pode ser considerada uma excelente matéria-prima para a produção de painéis. A maior dificuldade está na falta de interação direta com a maioria dos adesivos. Os resultados experimentais mostraram que, processando as partículas em moinho de martelos, demonstraram melhoras consideráveis na colagem interna, MOR e MOE dos painéis.

\section{CONCLUSÕES}

O acréscimo do percentual da casca de arroz nos painéis proporcionaram menores estabilidade dimensional e resistência mecânica. Painéis colados com tanino-formaldeído apresentaram qualidade superior àqueles em que foi utilizado ureia-formaldeído. Embora a utilização de casca de arroz tenha proporcionado uma redução nos parâmetros qualitativos dos painéis, com a adição de um baixo percentual de casca (até $10 \%$ ), pode ser possível a produção de chapas aglomeradas com propriedades físico-mecânicas similares àquelas confeccionados exclusivamente com partículas de madeira. Uma das principais limitações do uso da casca de arroz em painéis é a dificuldade de colagem das partículas. Recomenda-se a condução de testes com diferentes formulações de resinas ou tratamentos físico-químicos das partículas para melhorar a colagem.

\section{REFERÊNCIAS BIBLIOGRÁFICAS}

AJIWE, V.I.E. et al. A pilot plant for production of ceiling boards from rice husks. Bioresoure Technology, Texas, v. 66, n. 1, p. 41-43, Jan./Apr. 1998.

AMERICAN NATIONAL STANDARDS INSTITUTE - ANSI-A-208.1-87. Mat-formed wood particleboard. New York, 1987.

AMERICAN SOCIETY FOR TESTING AND MATERIALS - ASTM D-1037. Standard test methods for evaluating properties of wood-based fiber and particle panel materials. Annual Book of ASTM Standards, Philadelphia, v. 04.09, 1998.

CALEGARI, L. et al. Adição de aparas de papel reciclável na fabricação de chapas de madeira aglomerada. Ciência Florestal, Santa Maria, v. 14, n. 1, p. 193-204, jan./jun. 2004.

CALEGARI, L. et al. Desempenho físico-mecânico de painéis fabricados com bambu (Bambusa vulgaris Schr.) em combinação com madeira. Cerne, Lavras, v. 13, n. 4, p. 57-63, out./dez. 2007.

CARNEIRO, A. C. O. et al. Propriedades de chapas de flocos fabricadas com adesivo de ureia-formaldeído e de taninos de casca de Eucalyptus grandis W. Hill ex Maiden ou de Eucalyptus pellita F. Muell. Revista Árvore, Viçosa, v. 28, n. 5, p. 715-724, set./out. 2004.

CHEN, T. Y. Studies on the manufacture of particleboard from rice hulls in industrial scale. K'o Hsueh Fa Chan Yueh K'an, China, v. 8, n. 5, p. 456-462, Aug. 1980.

CINIGLIO, G. Avaliação da secagem de madeira serrada de Eucalyptus grandis e Eucalyptus urophylla. 1998.73 f. Dissertação (Mestrado em Ciência Florestal) - Universidade de São Paulo. Escola Superior de Agricultura Luiz de Queiroz, Piracicaba.

DACOSTA, L. P. E. et al. Qualidade das chapas de partículas aglomeradas fabricadas com resíduos do processamento 
mecânico da madeira de Pinus elliottii Engelm. Ciência Florestal, Santa Maria, v. 15, n. 3, p. 311-322, jul./set. 2005. FERREIRA, G. W. et al. Qualidade da celulose kraft-antraquinona de Eucalyptus dunnii plantado em cinco espaçamentos em relação ao Eucalyptus grandis e Eucalyptus saligna. Ciência Florestal, Santa Maria, v. 7, n. 1, p. 4163, jan./dez. 1997.

GERMAN STANDARDS COMMITTEE. Deutschen Normanausschuss. Specifications for particleboards. Holtz, 1971. (DIN 68761 (1)-1961 (3)).

HAN, G. et al. Upgrading of urea formaldehyde-bonded reed and whet straw particleboards using silane coupling agents. Journal of Wood Science, Tokyo, v. 44, n. 2, p. 282-286, Mar./Apr. 1998.

HANCOCK, W. V.; CHANDRAMOULI, P. Comparatives proprieties of rice-husk board, particleboard, and wafer board. Journal of the Indian Academy of Wood Science, India, v. 5, n. 1, p.18-27, Jan./June 1974.

HILLIG, E.; HASELEIN, C. R.; SANTINI, E. J. Propriedades mecânicas de chapas aglomeradas estruturais fabricadas com madeiras de pinus, eucalipto e acácia-negra. Ciência Florestal, Santa Maria, v. 12, n. 1, p. 59-70, jan./jun. 2002.

HIZIROGLU, S. et al. Properties of bamboo-rice straw-eucalyptus composite panels. Forest Products Journal, Madison, v. 55, n. 12, p. 221-225, Dec. 2005.

INSTITUTO BRASILEIRO DE GEOGRAFIA E ESTATÍSTICA - IBGE. Produção agrícola mundial: comentários. Rio de Janeiro: IBGE, 2005. 48 p. v. 35.

IWAKIRI, S. A influência de variáveis de processamento sobre propriedades de chapas de partículas de diferentes espécies de Pinus. 1989. 129 f. Tese (Doutorado em Engenharia Florestal) - Universidade Federal do Paraná, Curitiba.

KELLY, M. W. Critical literature review of relationships between processing and physical properties of particleboard. Madison: USDA Forest Service - FPL, 1977. 66 p.

LEE, H. H.; KANG, C. W. Development of rice hull insulation board using urea formaldehyde resin. Mokchae Konghak, China, v. 26, n. 4, p. 50-55, 1998.

LOPES, M. C. et al. Agrupamento de árvores matrizes de Eucalyptus grandis em função das variáveis dendrométricas e das características tecnológicas da madeira. Ciência Florestal, Santa Maria, v. 14, n. 2, p. 133-144, jul./dez. 2004.

MELO, R. R. Propriedades físico-mecânicas e resistência a biodeterioradores de chapas aglomeradas constituídas por diferentes proporções de madeira e casca de arroz. 2009. 77 f. Dissertação (Mestrado em Engenharia Florestal) - Universidade Federal de Santa Maria, Santa Maria.

NDAZI, B. et al. Production of rice husks composites with Acacia mimosa tannin-based resin. Journal of Materials Science, Storrs, v. 41, p. 6978-6983, Nov. 2006.

OKINO, E. Y. A. et al. Resistência físico mecânica de chapas aglomeradas de bagaço de cana-de-açúcar modificado quimicamente. Scientia Forestalis, Piracicaba, n. 52, p. 35-42, jul./dez. 1997.

PAULESKI, D. T. et al. Características de compósitos laminados manufaturados com polietileno de alta densidade (PEAD) e diferentes proporções de casca de arroz e partículas de madeira. Ciência Florestal, Santa Maria, v. 17, n. 2, p. 157-170, abr./jun. 2007.

PEDRAZZI, C. et al. Qualidade de chapas de partículas de madeira aglomerada fabricadas com resíduos de uma indústria de celulose. Ciência Florestal, Santa Maria, v. 16, n. 2, p. 201-212, abr./jun. 2006.

PIZZI, A.; SCHARFETTER, H.; KES, E. W. Adhesives and techniques open new possibilities for the wood processing industry. 1. Experience with tannin based adhesives. National Timber Research Institute, South Africa, v. 39, n. 3, p. 85-89, July/Sept. 1981.

SCHNEIDER, P. R.; SCHNEIDER, P. S. P.; SOUZA, C. A. M. Análise de regressão aplicada à Engenharia Florestal. 2. ed. Santa Maria: FACOS-UFSM, 2009. 294 p.

STATISTICAL ANALYSIS SYSTEM - SAS. Software computacional. Ambiente VM. Cary: New York, USA, Versão 6.08, 1998.

TEIXEIRA, D. E.; COSTA, A. F.; SANTANA, M. A. E. Aglomerados de bagaço de cana-de-açúcar: resistência natural ao ataque de fungos apodrecedores. Scientia Forestalis, Piracicaba, n. 52, p. 29-34, jul./dez. 1997.

TOMAZELLO FILHO, M. Variação radial da densidade básica em estrutura anatômica da madeira do Eucalyptus globulus, Eucalyptus pellita e Eucalyptus acmenioides. IPEF, Piracicaba, n. 36, p. 35-42, maio/ago. 1987.

YOUNGQUIST, J. A. et al. Agricultural fibers in composition panels. In: XXVII INTERNATIONAL PARTICLEBOARD/COMPOSITE MATERIALS SYMPOSIUM, 27., 1993, Washington. Proceedings... Washington: Washington State University: Pullman, 1993. p.133-152. 